\title{
Optimal \& Computationally Efficient Resilient Technique (OCERT) for Optical WDM Networks
}

\author{
Amit Kumar Garg \\ Electronics \& Communication Engg. Deptt. \\ Deenbandhu Chhotu Ram University of Science \& Technology \\ MURTHAL-131039, Sonepat (HR.) INDIA \\ garg_amit03@yahoo.co.in
}

\begin{abstract}
The survivability of Wavelength Division Multiplexing (WDM) networks is a very critical issue and there has been great research interest in the area of survivable WDM network design. Also, network service recovery from multiple correlated failures is a major concern given the increased level of infrastructure vulnerability to natural disasters, massive power failure and malicious attacks. Thus, this research paper has been framed considering protection and restoration mechanisms in order to guarantee the survivability of the networks. In this paper, for based optical networks, link and node failures recovery using p-cycles have been accounted for. The proposed optimal \& computationally efficient resilient technique takes care both node and link failure recovery relied upon a set of suitable candidate p-cycles for reducing the existing high computation time problem. The capacity efficiency and protection or restoration capabilities of proposed scheme are evaluated through numerical results. Extensive simulation results obtained indicate that the proposed scheme outperforms the traditional approaches in terms of performance metrics such as blocking probability, resource utilization efficiency and computational time.
\end{abstract}

Keywords: Network survivability, p-cycle, linear programming, blocking probability

\section{Introduction}

Optical networks are the communication systems which are based on single or multiple wavelengths of light for transmission. It is a promising technology for accommodating growing bandwidth demands. Optical networks are becoming an excellent choice as transmission systems for future backbone networks. Optical networks are able to provide high speed transport services to predominant client layers, such as Internet Protocol (IP), Asynchronous Transfer Mode (ATM) and Synchronous Optical Network (SONET) or Synchronous Digital Hierarchy (SDH). These services may cooperate with user applications. Several other layer combinations are possible such as IP over SONET over optical and ATM over optical [1]. In optical networks, Wavelength Division Multiplexing (WDM) has been developed as a technology that can satisfy the required bandwidth demand for the future transport networks [2]. Wavelength Division Multiplexing (WDM) is an important technique which allows the transport of large quantities of data over optical networks. However, due to the high traffic load that each link can carry in a WDM network, survivability against failures becomes very important. Survivability in this context is the ability of the network to maintain continuity of service against failures, since a failure can lead to huge data losses. Many survivability mechanisms have been studied and their performance is assessed through capacity efficiency, restoration time and restorability. Survivability mechanisms for ring and mesh topologies have received

Received (January 22, 2017), Review Result (July 7, 2017), Accepted (August 19, 2017) 
particular attention. The technique for survivability in optical networks is classified into two categories depending on how the spare capacity is allocated: pre-planned protection and dynamic restoration [2]. Pre-planned protection reserves backup paths against failure at the same time as the working paths are allocated. All working channels are dynamically switched to reserved paths in the case of failure. Automatic Protection Switch (APS), Self Healing Ring (SHR) network mechanism and Mesh network mechanism belong to preplanned protection since these schemes allocate backup paths in advance. This protection technique is able to offer fast computation time since backup paths are pre-computed and the nodes on the working path only need to be changed to the nodes on the backup path. However, capacity utilization is a major challenge in these protection schemes, except for the mesh network mechanisms. Unlike the other two schemes, the mesh mechanism offers efficient capacity utilization, but has higher computation time. Research has shown that path protection offers better capacity utilization but link protection offers faster restoration [3-4]. In terms of capacity allocation strategies, survivability schemes can be classified as dedicated protection or shared protection. In dedicated protection schemes, signals can be simultaneously transmitted on working routes and backup routes, while signals on shared protection schemes are only transmitted on working routes and switched to backup routes when a failure occurs. Thus, the dedicated schemes offer faster restoration but require much more spare capacity compared to shared protection schemes. A p-cycle is about pre-configured protection cycles in a mesh network and it merges the strengths of ring and mesh topologies; i.e. the fast recovery speed of ring restoration and the capacity efficiency of mesh protection. A p-cycle is oriented, ensures a fast restoration time (typically of the order of 50 to $80 \mathrm{~ms}$ ) and p-cycles are preconfigured [5]. A p-cycle does not traverse a node or a link more than once. The p-cycle protection approach can achieve an efficient use of the network capacity compared to other protection approaches, such as the one-plus-one $(1+1)$ and the one-by-one (1:1) restoration methods [6]. In fact, a p-cycle can protect both on-cycle links and straddling links. An on-cycle link belongs to the p-cycle, and is directed opposite to the p-cycle. A straddling link does not belong to a p-cycle. However, its extremity nodes are traversed by the p-cycle. The p-cycles allow the required backup bandwidth capacity to be reduced. The factors that will influence the applicability of p-cycles include the nodal degree, network topology, network size, network capacity, wavelength utilization and traffic load. The survivability of a network refers to a network's capability to provide continuous service and maintain quality of service in the presence of such failures. Survivability can be classified into three categories:1) prevention, 2) network design, 3) traffic management and restoration [4-6]. Prevention focuses on improving equipment and system reliability. Network design aims to achieve efficiency in terms of sufficient spare capacity and faster restoration time against failures. Traffic management and restoration are concerned with the network load in the event of failure and connection re-establishment around the failure. Survivability should guarantee maximum restorability to provide quality of service (QoS) against failures. There exists both pre-planned protection and dynamic restoration mechanisms in survivability. Dynamic protection methods are not able to guarantee $100 \%$ protection, but offers faster restoration time [4].Pre-planned protections, however, provide 100\% protection by reserving alternative paths in advance [5]; hence this mechanism is a more interesting development in survivable networks. Existing approaches for solving the pcycles problem include Integer Linear Programming (ILP) algorithms. ILP requires a set of candidate p-cycles to be pre-computed. It is usually easy to find the optimal solution for small or medium size networks because of the small number of possible cycles. Thus, finding a subset of candidate p-cycles to improve the performance is an important issue [6].

The rest of this paper is organized as follows. In Section 2, background and related work comprising of various techniques to ensure protection and restoration mechanisms has been discussed for deployment of reliable optical WDM based network. In Section 3, 
a novel survivable scheme has been proposed which is both optimal and computationally efficient Resilient Technique (OCERT) for Optical WDM mesh networks. Extensive simulations and numerical results are presented in Section 4 . The conclusions followed by future expansions are given in Section 5.

\section{Background and Related Work}

Optical WDM networks are required to satisfy the growing network traffic demands. Therefore, survivable network design against failure in WDM network becomes a critical issue since a channel failure can lead to huge data losses. Automatic Protection Switching is one of the attractive schemes to provide satisfactory quality of service in the networks [3-4]. The APS can be divided into three different architectures depending on the assignment of protection resources: 1+1 APS, 1:1 APS and 1: N APS. These three models select backup path after link failures. 1+1 APS is a sort of dedicated protection, 1:1 APS and 1: N APS are shared protection. These techniques offer efficient capacity utilization but have high blocking probability. In optical networks, a ring topology, known as Self Healing Ring (SHR), is the simplest protection network when a protected link fails. SHR easily provides better restorability and offers faster computation time because of simple control management and simple routing policy. In SHR, half of total capacity is reserved for working capacity while the other half is used as spare capacity. Upon a failure of a single link, the working channel is rerouted along a backup channel in the opposite direction. The existing protection schemes suffer from imbalance between capacity utilization and computation time. The mesh topology is preferred for large networks while ring topology is for simple and small-scale networks. In mesh network survivability, it is more complicated to solve the optimization problem since a higher number of routing and wavelength channels must be considered. But, mesh network survivability offers high capacity utilization because spare capacity can be shared to protect against network failures so that this network mechanism is preferred more from an efficiency point of view. Mesh network based survivability is classified into two categories depending on the re-connecting process: path protection and link protection. Path based survivability is more efficient in capacity utilization compared to link based survivability, since it only needs spare capacity for the whole reserved path instead of every link along the path. In link based survivability, restoration time is faster than path based survivability since path mechanism requires a longer time to generate a fault notification message. Dynamic restoration dynamically discovers backup paths in the network after a link fails. Typically, this is more efficient in term of capacity utilization than pre-planned protection since it is not necessary to reserve spare capacity. On the other hand, computation time is a challenging problem to tackle in dynamic restoration. This mechanism can be classified into path restoration and link restoration [7]. Path restoration is more efficient than link restoration in terms of spare capacity utilization where as link restoration is faster than path restoration [8]. Shared Backup Path Protection (SBPP) is similar to the $1+1$ APS scheme in it two disjoint paths, a working and backup path are routed to transmit the optical signal. SBPP is one of the survivability techniques which offers great capacity efficiency, but with a slow computation time. Pre-configured protection cycle (p-cycle) is considered as link protection in mesh networks. The p-cycle method is based on closed cyclic routes. The main focus of p-cycles is to recover failures using ring network mechanism in a mesh network. This mechanism is a promising approach for solving the network design problem in the context of survivability, because p-cycles can achieve a fast restoration time like ring protection and a high efficiency capacity like mesh protection [9]. A p-cycle achieves fast restoration times since only the nodes adjacent to the failure perform protection switching. The high capacity efficiency is due to the fact that a p-cycle can provide protection not only for on-cycle links but also for straddling links without requiring any additional spare capacity. A straddling link is a link which 
does not belong to the p-cycle but whose end-nodes are both on the p-cycle. This property effectively reduces the required protection capacities. The efficiency of a p-cycle is inversely proportional to its redundancy. The redundancy [10] of a cycle is defined as the ratio between the spare capacity (number of wavelengths used by the cycle) and the working capacity of the cycle (number of wavelengths protected by the cycle on its oncycle and straddling links). A p-cycle with high efficiency is a p-cycle that has a small value of redundancy, in other words it protects more working wavelengths using fewer spare wavelengths. This technique is a reliable and effective method for utilization of capacity in optical mesh networks [10-11]. However, determination of the optimal set of p-cycles for protection is an NP-hard problem. A p-cycle protection offers useful restoration paths depending on the relationship to the failed link. Searching suitable paths is an important issue in p-cycle protection since efficient paths can offer better capacity efficiency. Existing approaches for solving the p-cycle problem are through the use of an Integer Linear Programming (ILP) model or using heuristic methods [12-13]. The main problem with ILP is that it requires all possible cycles, which can be a very large number in dense and large scale networks. Thus, the computational complexity in ILP formulation is a complex issue. The heuristic approach based on ILP can achieve near optimal solutions, but it is still an optimization problem with the associated computational complexity of ILP. Computational complexity and optimality of solutions are two performance metrics which pose conflicting requirements in optimization problems [14$15]$.

In the present work, for WDM based optical networks, link and node failures recovery using p-cycles have been considered. The proposed OCERT scheme ensures node and link failure recovery based on a set of suitable candidate p-cycles to overcome the high computation time problem.

\section{Proposed Optimal and Computationally Efficient Resilient Technique (OCERT) for Optical WDM Networks}

Computational complexity is an important factor when evaluating an algorithm. It is defined as the time it takes for an algorithm to find a solution. The ILP model suffers from high computational complexity in dense networks even though it computes the optimal solution. If an algorithm can achieve a near optimal solution within an acceptable computational complexity, it will likely be a more desirable solution to the problem. The proposed technique investigates the possibility of using a heuristic method in order to achieve the best performance in terms of computational complexity. Computation time and the capacity utilization are managed by the proposed scheme to achieve the desired restorability. Consequently, it ensures better protection while minimizing the total spare capacity and reducing computation time. The following is the detailed description of analytical model of the proposed OCRET scheme for WDM systems.

1. A physical topology is modelled as a connected graph $\mathrm{G}(\mathrm{N}, \mathrm{L})$ where $N$ is the set of network wavelength routing nodes and $L$ is the set of network single-fiber.

2. The proposed OCERT scheme first finds the shortest path between two end nodes of a span and then searches other shortest path between the same node-pairs that are node disjoint from the previous path. It then constructs a cycle by combining the two shortest node-disjoint paths (each path contains no common intermediate nodes).

3. The main motive of using proposed scheme (OCERT) is to offer protection in mesh optical networks by combining the benefits of ring like recovery speed and the efficiency of a mesh network, however the concept is not only limited to transport optical networks and can be extended to higher levels and other network types such as optical multicast media traffic. 
4. It is seen that creating p-cycles is computationally intensive when the number of nodes is large. The Heuristic method presented called the ER-based unity-p-cycle, shows an attractive solution to solve the problem with creating p-cycles without the use of ILP. This method also has a solution that is close to that of an optimal solution, but without the extra computational time required. The main focus of this scheme is to identify unity p-cycles that are able to protect as many working links as possible; this essentially reduces the number of spare units required for protection. A unit p-cycle is able to protect one working link in opposite direction for every on cycle span and two working units for every straddling span. The number of spare units of a unity p-cycle is equal to the number of the spans on the cycle. A ratio called ER is defined as the number of working links that are protected by the unity p-cycle to the number of spare units. The higher the ratio the better the efficiency of the protecting p-cycles and the same is achieved by the proposed OCERT scheme. The brief description of the proposed scheme is shown below:

a) Search for the possible cycles and determine the working capacity for each based on one of the shortest path.

b) Calculate the ratio ER of the unit-cycles for the cycles as per step 1 .

c) Based on the ER calculation select the cycle with the highest ER.

d) Remove the working links that can be protected by the selected cycle from above \& update the working capacity.

e) Repeat the above steps until the working capacity on every span is 0 .

5. In the proposed scheme, the total traffic request is known in-advance and the connection is dynamically selected dependant on the current network state such as traffic patterns and channels available i.e. the link which has enough available wavelengths is first considered to establish a connection. For dynamic wavelength assignment, heuristic approach is used to assign wavelengths to route a connection. The proposed scheme is a two-step process which first determines all possible cycles in the network and then selects a set of suitable candidate cycles. All on-cycles are organized in ascending order of the hop length. An on-cycle is a small cycle which contains the span itself but does not contain any straddling link. In order to reduce the number of candidate $p$-cycles by computing high efficiency cycles, a 'pre-selection' heuristic approach has been used. Thus, a high efficiency cycle is defined as a cycle with high potential capability to protect against network failure.

6. The brief working of proposed Scheme employing heuristic scheme is as follows:

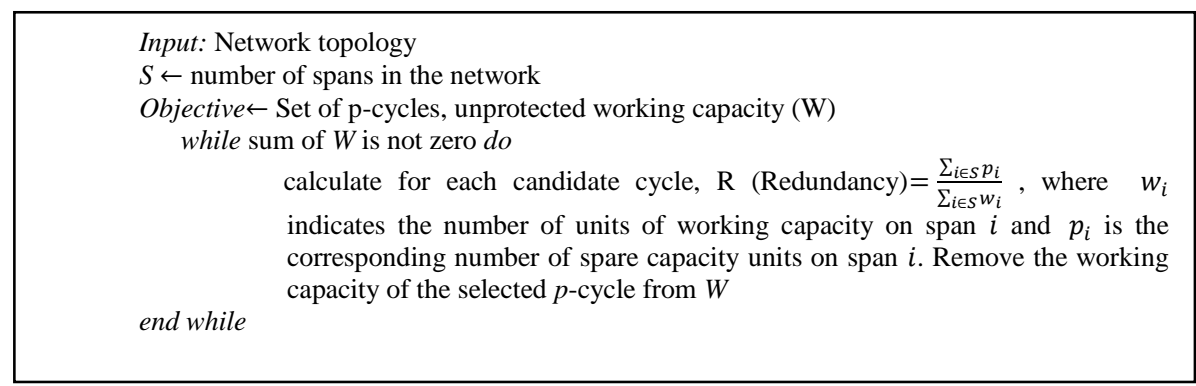

7. The following is the functional description of the model of the proposed OCERT scheme. 


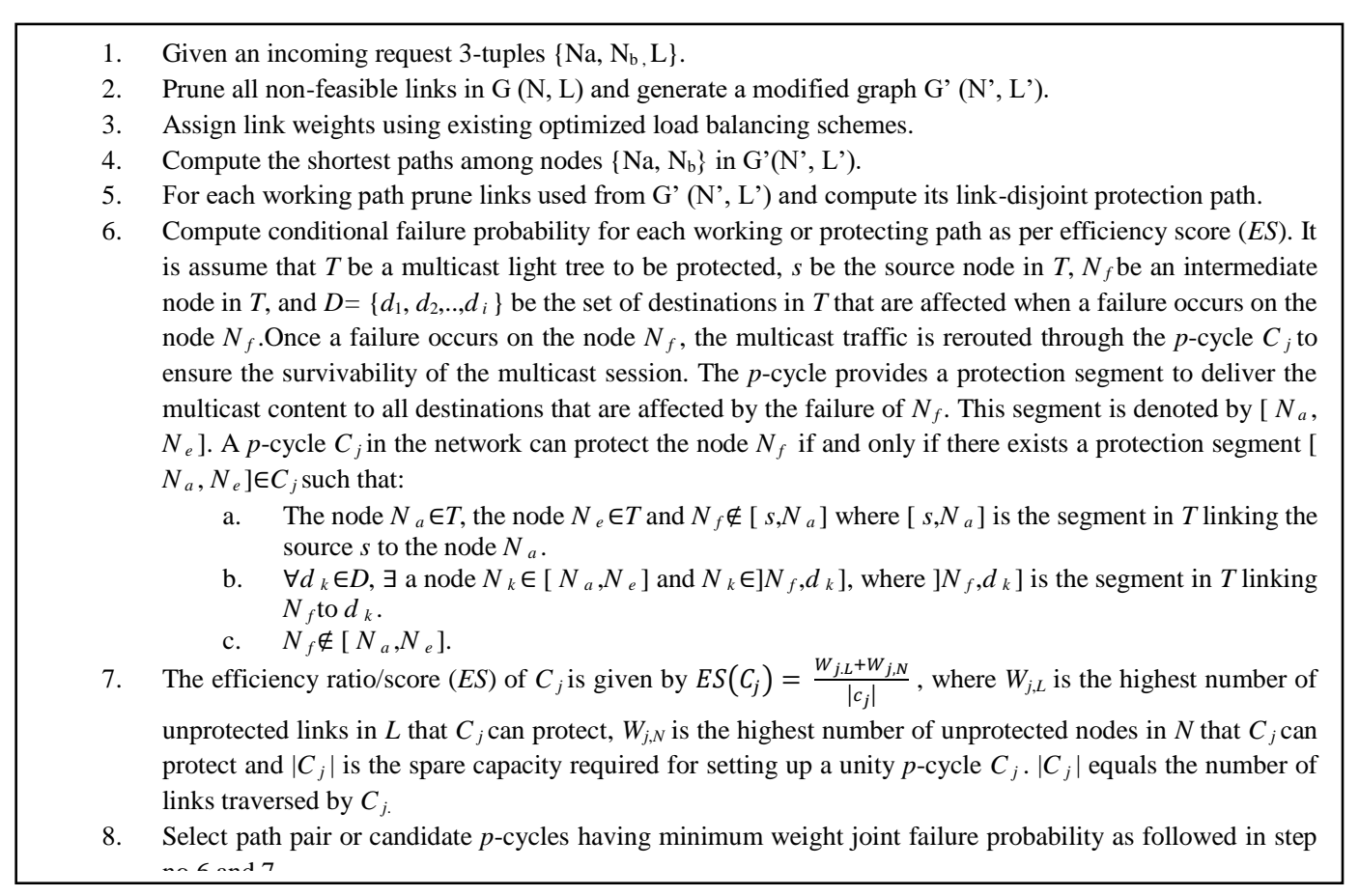

After routing a multicast tree, the set of links in $L$ and the set of nodes in $\mathrm{N}$ have been computed that can be protected by the existing p-cycles in the network. Also, the reserved capacity of an existing p-cycle in the network is released when the p-cycle does not protect any working link and nodes in the network. Therefore, the proposed OCRET scheme ensures the restoration based on a set of suitable candidate p-cycles to overcome the high computation time problem.

\section{Performance Evaluation}

The proposed OCERT scheme guarantees the recovery from link and node failure in WDM based optical networks with a fast restoration time. It has been compared with the traditional approaches which were reported to be the most efficient techniques for dynamic traffic protection in terms of resource utilization efficiency and blocking probability. The bandwidth required for each connection request is function of the modulation format which is proportional at transmission path length and the signal propagation speed on a unit of bandwidth and connection speeds. Also in the simulation, it is assumed that request arrival follows a poisson process with an average arrival rate $\lambda$ and the request holding time follows an exponential distribution with an average holding time $\mu$. Thus, the offered traffic load for the network is given by $\lambda \mu$. The results for network traffic simulations have been obtained using the software Network Simulator [16] and for analytic results, data processing and plotting are carried out using standard commercial software. The backup route is the shortest hop-length path that is link-disjoint to the route of the original lightpath. It is assumed that the original lightpath establishment is on the route with the minimum number of hops between source-destination nodes, i.e., the route with the shortest hop length. The processing delay for handling each restoration request on each node is assumed to be equal to 6 microseconds. Link transmission rate is $2.5 \mathrm{~Gb} / \mathrm{s}$. Each node maintains global network state information for routing and this information is periodically updated. The network topology used in the simulation is shown in Figure 1, which has 7 nodes, 13 links, and an average nodal degree of 3.7143 has a total of 58 possible cycles (whereas the traditional NSFNET topology (14nodes, 21 links,) has 139 possible cycles). 




Figure 1. Simulated Network Topology

Without loss of generality, it is assumed that each link has two fibers. The two fibers transmit in opposite directions; 16 wavelengths are available on each fiber. The source and the destinations of each multicast session are randomly selected (uniform distribution law). The number of destinations in each multicast request equal to 5, which seems to be reasonable as the total number of nodes in the used topology is less than 16.The performance metrics used for evaluation, is blocking probability, resource utilization and average computation time. The blocking probability is the percentage of requests that cannot be routed or protected among the total number of requests. The resource utilization is the percentage of reserved wavelengths in the network among the total number of wavelength links. The average computation time is required for routing and protecting a traffic request. A performance criterion for each metric is computed according to the traffic load. For each traffic load value, $5 \times 10^{5}$ requests were generated. This number of requests is enough to measure blocking probability, resource utilization and average computation time with a $95 \%$ confidence interval. The proposed OCERT scheme is simulated (as shown in Figure 2) with two different values for the number of candidate pcycles (800 and 400). For the existing techniques, the blocking probability increased when the traffic load was high. It is seen that the proposed OCERT scheme outperforms the existing techniques and has a lower blocking probability, especially when the traffic load was high. The proposed scheme with number of candidate p-cycles as 800 has the lowest blocking probability. When number of candidate p-cycles is 400 , the blocking probability of proposed scheme increased but remained lower than that of traditional ones. This is because as number of candidate p-cycles (400) is very low compared to the total number of p-cycles in the existing network (NSFNET). 


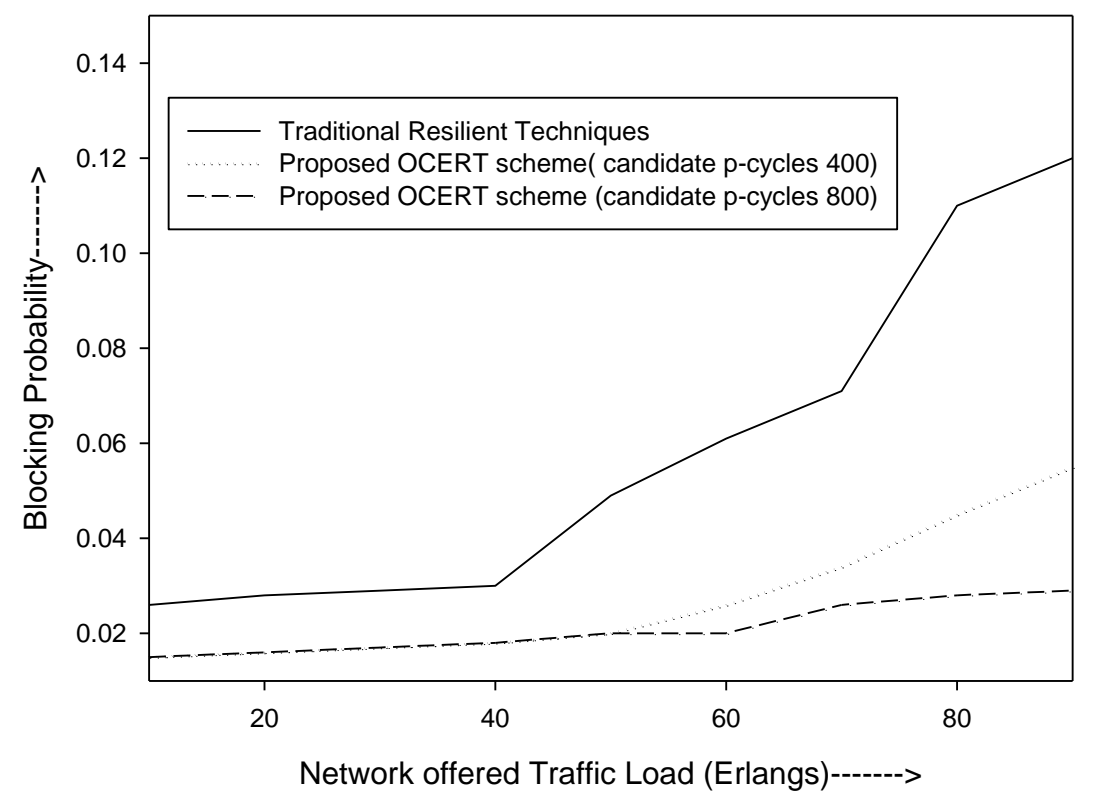

Figure 2. Performance Comparison of Blocking Probabilities



Figure 3. Performance Comparison of Resource Utilization

The resource utilization of various schemes has been compared as shown in Figure 3. When the traffic load increases, the wavelength percentage reserved per link is higher for both proposed OCERT scheme as well as traditional schemes. The wavelength percentages reserved by the proposed scheme for number of candidate p-cycles (800 and 400) are very close. This percentage is very low compared with that of the traditional ones, especially when the traffic load is high. For a traffic load equal to 65 erlangs, almost $70 \%$ of the wavelengths on each link are reserved for the proposed scheme and $80 \%$ for the traditional ones.

To assess the speed of the proposed scheme, performance metric average computation time has been considered for setting up a multicast request. The average computation time in the proposed OCERT scheme with both candidate p-cycles is very low compared with 
that of the existing scheme (as shown in Figure 4). This is due to the availability of lower number of p-cycles for the protection.

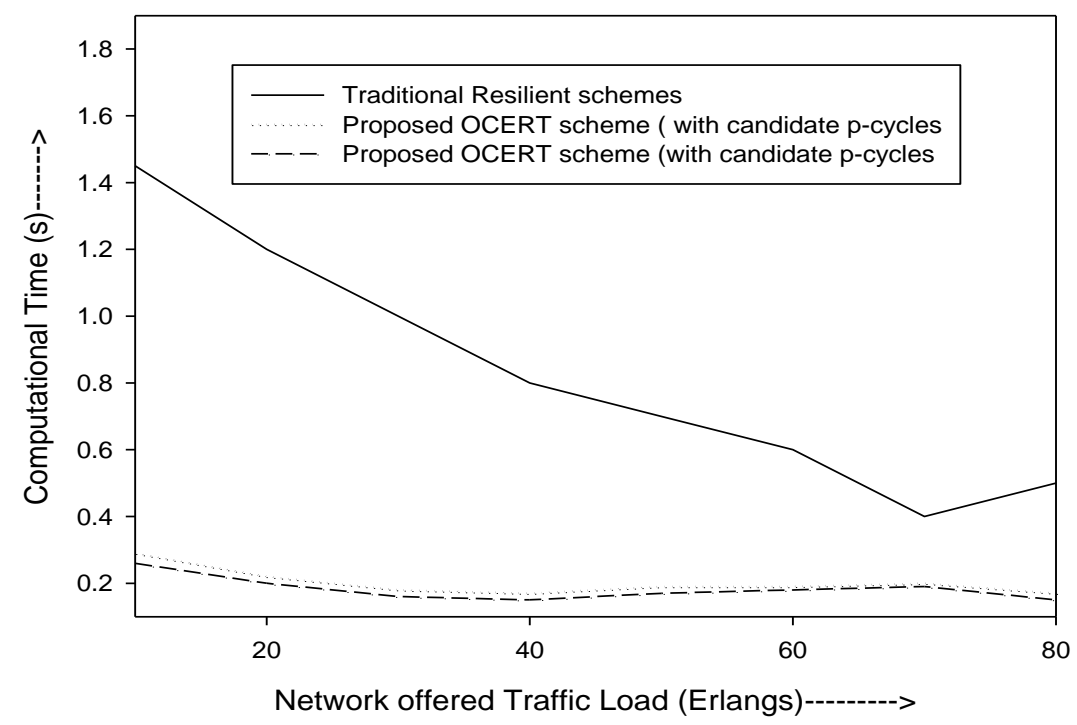

Figure 4. Performance Comparison of Average Computation Time

Table 1. Performance Comparison of Proposed OCERT Scheme

\begin{tabular}{|l|c|c|}
\hline \multirow{2}{*}{ Attributes } & \multicolumn{2}{|c|}{ Network Topology } \\
\cline { 2 - 3 } & Simulated topology (7-nodes) & NSFNET ( Existing with 14 nodes) \\
\hline Maximum no. of cycles found & 58 & 139 \\
\hline Average cycle length & moderate & large \\
\hline Average Computation time (sec) & low & high \\
\hline No. of Shortest paths for end nodes & 12 & 22 \\
\hline
\end{tabular}

The results as shown above in Table. 1 indicate that the proposed OCERT scheme finds all possible cycles within a reasonable time in comparison with existing ones.

\section{Conclusion \& Future Scope}

In the present work, survivability algorithms based on p-cycles in optical WDM mesh networks have been investigated with a motive on balancing towards optimality of solution and computational complexity. The proposed OCRET scheme ensures node and link failure recovery based on a set of suitable candidate $p$-cycles to overcome the high computation time problem. The simulation results obtained indicate that the proposed OCERT scheme outperforms the traditional schemes in terms of blocking probability, resource utilization and computation time. The simulation results confirms the effectiveness of the proposed scheme however in future some other optimization techniques and requirements parameters may also be analyzed for reducing the calculation time. Also, future efforts will look at developing more detailed optimization models to derive lower bounds on achievable performance. 


\section{References}

[1] H. Zhang and O. Yang, "Finding protection cycles in DWDM networks", Proceedings of the International Conference on Communications (ICC), New York, USA, (2002).

[2] S. Ramamurthy, L. Sahasrabuddhe and B. Mukherjee, "Survivable WDM mesh networks", Journal of Lightwave Technology, vol. 21, no. 4, (2003), pp. 870-877.

[3] P. Ho, J. Tapolcai and H. T. Mouftah, "On achieving optimal survivable routing for shared protection in survivable next-generation Internet", IEEE Transactions on Reliability, vol. 53, no. 2, (2004).

[4] Z. Zhang, W. D. Zhong and S. K. Bose, "Dynamically survivable WDM network design with p-cycle based PWCE", IEEE Communication Letters, vol. 9, (2005), pp. 756-758.

[5] R. Inkret, M. Lackovic and B. Mikac, "Applying p-cycles to multiservice photonic network", Proceedings of 7th Int. Conf. on Transparent Optical Networks, Barcelona, Spain, (2005).

[6] D. A. Schupke, "Analysis of p-cycle capacity in WDM networks", Photonic Network Communication, vol. 12, no. 1, (2006), pp. 41-51.

[7] Q. Liu, M. Ko, N. Ghani and A. Gumaste, "Hierarchical routing in multi-domain optical networks", Computer Communication, vol. 30, no. 1, (2006), pp. 122-131.

[8] R. Asthana and Y. N. Singh, "Second phase reconfiguration of restored path for removal of loop back in p-cycle protection”, IEEE Communication Letters, vol. 11, no. 2, (2007), pp. 201-203.

[9] J. Berthold, A. A. M. Saleh, L. Blair and J. M. Simmons, "Optical Networking: Past, present and future”, Journal of Lightwave Technology, vol. 26, no. 9, (2008), pp. 1104-1118.

[10] R. Asthana and Y. N. Singh, "Distributed protocol for removal of loop backs and optimum allocation of p-cycles to minimize the restored path lengths", Journal of Lightwave Technology, vol. 26, no. 5, (2008), pp. 616-627.

[11] J. Szigeti, R. Romeral, T. Cinkler and D. Larrabeiti, "p-Cycle Protection in Multi-Domain Optical Networks", Photonic Network Communications, vol. 17, no. 1, (2009), pp. 35-47.

[12] A. E. Kamal, "1+N Network Protection for Mesh Net-works: Network Coding-Based Protection Using p-Cycles”, IEEE/ACM Transactions on Networking, vol. 18, no. 1, (2010).

[13] M. F. Habib, M. Tornatore, M. De Leenheer, F. Dikbiyik and B. Mukherjee, "Design of DisasterResilient Optical Datacenter Networks", Journal of Lightwave Technology, vol. 30, no. 16, (2012).

[14] H. Alazemi, S. Sebbah and M. Nurujjaman, "Fast and efficient network protection method using path pre-cross-connected trails", Journal of Optical Communications and Networking, vol. 5, no. 12, (2013), pp. 1343-1352.

[15] J. Lopez Vizcaino, "Protection in optical transport networks with fixed and flexible grid: cost and Energy efficiency evaluation”, Optical Switching and Networking, vol. 11, (2014), pp. 55-71.

[16] The Network Simulator: NS2, http://www.isi.edu/ ns-nam /ns/. 